
\title{
$\begin{array}{ll}\text { Research Square } & \begin{array}{l}\text { Preprints are preliminary reports that have not undergone peer review. } \\ \text { They should not be considered conclusive, used to inform clinical practice, } \\ \text { or referenced by the media as validated information. }\end{array}\end{array}$
}

\section{The jump and stagnation of mass with speed}

\section{Dong Jun ( $\sim$ dongjun19@nuaa.edu.cn )}

Nanjing University of Aeronautics and Astronautics https://orcid.org/0000-0002-6289-2572

\section{$\mathrm{Na}$ Dong}

Southeast University

\section{Article}

Keywords: Special and general relativity, Coordinate transformation in the flat space of Riemannian geometric space, Instantaneous local inertial coordinate system, Relationship between mass and speed, Step function relationship of mass with speed

Posted Date: May 7th, 2021

DOl: https://doi.org/10.21203/rs.3.rs-489062/v1

License: (c) (i) This work is licensed under a Creative Commons Attribution 4.0 International License. Read Full License

Version of Record: A version of this preprint was published at IET Quantum Communication on March 25th, 2022. See the published version at https://doi.org/10.1049/qtc2.12038. 


\title{
The jump and stagnation of mass with speed
}

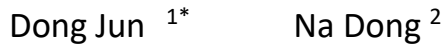 \\ $1^{*}$ (Nanjing University of Aeronautics and Astronautics College of Science Nanjing 210016, China) \\ ${ }^{2}$ (Southeast University School of Electronic Science and Engineering Nanjing 210096, China)
}

\begin{abstract}
In the general theory of relativity the four-dimensional space-time described the accelerated motion or moves in a gravitational field of a mass body, from the perspective of integral geometry, although it is a curved Riemannian geometric space, but for any instantaneous position of the moving mass body, there is a local Flat Space of Riemannian geometric space. The local Flat Space is a Mincowski space in which the inertial coordinate system can be used in the local small area. Between the proper coordinate systems of two interacting moving masses, or between a series of follow-up proper coordinate systems experienced by a mass body moving in any way, there must be a coordinate transformation relationship similar to the traditional special theory of relativity. However, they have an important difference: In these instantaneous local inertial systems, the speed of light is no longer the constant $c$ of vacuum, the effect of gravitational field or acceleration on the speed of light is the same as that of a medium with a dielectric constant of $\varepsilon$ and a permeability of $\mu$. Using the special theory of relativity with variable speed of light that the author has established can discuss relevant relativity physics issues in these instantaneous local inertial systems. This article uses the special theory of relativity with variable speed of light to infer the functional relationship between a moving mass and the change of speed. In addition to obtaining the traditional continuous increasing function relationship, a step function relationship with stepped discontinuous changes is also obtained. At the same speed, the mass can have two values, such as a ladder upgrade one level; the same mass can be matched with two different speeds, such as one step extension forward on the same step stair. From the perspective of the increase in speed, the mass is stagnant on the step platform (the speed increases, the mass does not change), and it jumps in the step up ladder (the speed does not change, the mass has a jump change). This obviously incorporates the main image of quantum theory into the theory of relativity, which is the result that all physics researchers care about and expect.
\end{abstract}

Key words: Special and general relativity; Coordinate transformation in the flat space of Riemannian geometric space; Instantaneous local inertial coordinate system; Relationship between mass and speed; Step function relationship of mass with speed

\section{Introduction}

The mass of a moving body is a continuous increasing function of speed. This is an important conclusion of the traditional special theory of relativity, which shows that

\footnotetext{
$1 *$ e-mail: dongjun19@nuaa.edu.cn
} 
classical mechanics only has some approximations under the test of relativity. Unfortunately, the continuity of this mass-speed relationship is incompatible with all quantum theories. This becomes a major obstacle to trying to dominate modern physics with the theory of relativity. In fact, in addition to the conventional continuous change of the mass-velocity relationship in the theory of relativity, there is also a stepped, discontinuous change relationship. At the same speed, the mass can have two values, such as a ladder upgrade by one level; the same mass can be matched with two different speeds, such as a step extension forward. From the perspective of the increase in speed, the mass is stagnant on the step platform (the speed increases, the mass does not change), and it jumps in the step up ladder (the speed does not change, the mass has a jump change). This obviously incorporates the main image of quantum theory into the theory of relativity, which is the result that all physics researchers care about and expect. However, it is impossible to infer this conclusion with the traditional special theory of relativity, but it is easy to use the "variable speed of light" special theory of relativity established by the author of this article ${ }^{[1]} \cdot{ }^{[2]}$.

There is only one core theory of the theory of relativity, and that is: the mathematical form of the laws of physics should not differ depending on the observation coordinate system used. The traditional special theory of relativity is limited to the covariant of mathematical forms of physics laws between inertial coordinate systems in a vacuum; General relativity removes the restriction on the observation coordinate system and discusses the covariant of the mathematical forms of the laws of physics in the gravitational field or between accelerated and decelerated coordinate systems. General relativity applies four-dimensional Riemannian geometry to combine the physical quantities in the laws of physics into scalars, vectors, tensors, and higher-order tensors in a four-dimensional geometric space individually or with each other, and then according to the mathematical relationship between physical quantities established in physics, the relationship between scalar, vector and tensor in geometry is established. Geometry is deduced and demonstrated by a coordinate system, but the geometry proved by deduction does not differ depending on the selected coordinate system. In this way, the mathematical equation connecting these physical quantities has a very simple and symmetrical covariant form. This is the mainstream relativity work of the Classical Academy. If this work develops smoothly, it should not be out of touch with this aspect, that aspect, or any aspect of modern physics. Unfortunately, although the mathematical form of the laws of physics has a very symmetrical and beautiful covariant form in the mathematics tower of fourdimensional Riemannian geometry; however, it is very difficult to solve, apply, and even use. Einstein focused on the fundamental difference between "inertia" and "gravitational field existence", go all out to explore the difference between space and time structure in Riemannian geometric expressions, and uncontrollably transform what should be called general relativity into the study of gravitational field and gravitational field equations. In the calculation of the structure of mathematics to relieve the spatial structure of the gravitational field, only the metric tensor $g_{\mu \nu}$ and its first derivative $\frac{\partial g_{\mu v}}{\partial x^{\lambda}}$ can be taken care of, they appear in the curvature tensor 
$R_{\mu \nu}, R$ and affine connection (Christoffel symbols) $\Gamma_{\mu \nu}^{\lambda}$; for the second and higher derivatives of the basic metric tensor $g_{\mu \nu}$ to the coordinate $x^{\lambda}$, the "approximation" steps that are generally accepted in mathematics are omitted. In fact, this does not necessarily mean that a function is written as a convergent series in a serious mathematical way, which approximates the numerical problem of calculating this function. Serious mathematics must look at the mathematical steps with the known "boundary conditions" in real physics. Ignore the "boundary conditions" and use mathematics, or use generally recognized mathematical methods to deal with real physics problems that have not yet obtained "boundary conditions". The problem is that mathematics sets a tone first for physics theory. When the swing reaches its highest position, the first derivative of its circle displacement to time is zero, but isn't the second derivative value very large? Therefore, certain types of higher-order derivatives that are omitted due to insufficient "boundary values" are likely to be the true focus of the evolution of certain types of cosmological problems, atomic physics and even nuclear physics.

In general relativity, from the perspective of integral geometry, although the fourdimensional space-time that describes the accelerated motion of the mass body or the motion in the gravitational field is a curved Riemannian geometric space, but for any instantaneous position of the moving mass, there is a flat space of Riemannian geometric space. The flat space is Euclidean space, a Mincowski space, in which the inertial coordinate system can be used in a small area. There must be a coordinate transformation relationship similar to the special theory of relativity between the proper coordinate systems of two interacting moving masses, or between a series of follow-up proper coordinate systems experienced by a mass moving in any way. However, they have an important difference: with acceleration or in a gravitational field, it is the same as in a medium, the speed of light is not a constant $c$ in vacuum, but another constant $c^{\prime}{ }^{[3]}$. Therefore we must face the problem of coordinate transformation between instantaneous local inertial coordinate systems with different speeds of light as the limit speed values. After in-depth thinking, the author of this article believes that If we can establish such a coordinate transformation between two instantaneous local inertial coordinate systems, we can lead the stagnant and inconvenient general theory of relativity away from the complex mathematical structure simply and successfully, so that the work of the theory of relativity to explore the physics laws that is invariable in mathematical form becomes easy.

In reference [1] and [2], the established "special theory of relativity in different media" is "the special theory of relativity with variable speed of light" which takes different speed of light as the limit value. Apply it to an instantaneous local inertial coordinate system on the tangent plane at any point in the curved Riemannian geometric space in general relativity, in fact, the general relativity is deduced into a convenient application form of the special theory of relativity. This article applies the reconstructed theory of relativity to relativistic mechanics and obtains an important conclusion: the change of the mass of motion with the speed, in addition to the 
traditional continuous increasing function relationship, there is a new discontinuous step function relationship. In the article, firstly, the transformation between accelerating coordinate systems or the coordinate system in the gravitational field in general relativity is processed as the coordinate systems transformation between two instantaneous local inertial coordinate systems with different speeds of light as the limit speed value which is at each point of the "flat space" in the four-dimensional Riemann geometric space. Then, two functional relations of the moving mass change with speed are derived: continuous increasing function and discontinuous step function, they do not coexist. Finally, the results are discussed and prospected.

\section{Coordinate transformation between instantaneous local inertial systems}

The purpose of general relativity is to derive the covariance of the laws of physics for non-inertial frames in accelerated motion and gravitational fields. Although the application of four-dimensional Riemannian geometry can combine the physical quantities in the laws of physics into vectors, tensors, and higher-order tensors that are covariant with the coordinate system in the four-dimensional geometric space, so the mathematical equations connecting these physical quantities have a very simple and symmetrical covariant form, but its calculation, application and utilization are very difficult. It is a work that Einstein has worked hard and failed to complete in the latter half of his life. ${ }^{[4]}$ Einstein's focus is on the fundamental difference between "inertia" and "gravitational field existence", and goes all out to explore the differences in the Riemannian geometric expressions of their respective space and time structures. The content that should really be called general relativity uncontrollably transformed into the study of gravitational field, this is easy to make people misunderstand that general relativity is a theory that specializes in the study of gravitational fields and cosmology, which is far from the original purpose of relativity. In fact, according to the principle of equivalence, every point in the space-time four-dimensional Riemann space describing the gravitational field or the accelerated motion has an instantaneous local inertial coordinate system. The trajectory of a mass point in arbitrary motion is a continuous or approximately continuous curve in space. "Approximately" means that if the point experienced in turn are discontinuous "points" ,connect these points into a curve as a trajectory. The vertical line of the contact surface of this curve and the tangent line and normal line on the contact surface can according to "forward" advanced in sequence of the mass point, and "left-handed" or "right-handed" on the tangent line and the system is "clockwise" or "reverse time", determine a mutually orthogonal follow-moving "proper coordinate system" three-dimensional space ( $x, y$, z), (or take "spherical coordinates", "cylindrical coordinates"), and the moving mass point is always located at the origin of the coordinate system. If you agree with the view of integral geometry, the four-dimensional space composed of the threedimensional space of the real physical world plus the "one-dimensional time" is a curved space installed in more than four-dimensional European space, based on the tangent line and the contact surface plus "time one-dimensional" to determine its follow-moving "proper coordinate system" constitutes a Euclidean folded flat space of four-dimensional space suitable for the application of differential geometry. In terms 
of geometry, such a flat space is all equivalent to a Minkowski space in which an inertial coordinate system can be used in a small instantaneous local area. So at the view point of a small instantaneous local area this kind of "proper coordinate system" is also an inertial coordinate system. ${ }^{[1]}$ Because in general relativity the speed of light is no longer the constant $c$. It has been proved that in the gravitational field or when there is acceleration, just like in the medium, the speed of light is no longer the constant $c$ in vacuum, but another constant $c^{\prime} .{ }^{[3]}$ The influence of gravitational field or acceleration on the speed of light is the same as that of a medium with a dielectric constant of $\varepsilon$ and a magnetic permeability of $\mu$. In the static gravitational field with unit mass gravitational potential energy $\chi$, the speed of light is $c^{\prime}=\frac{c}{n}=\frac{c}{\sqrt{\varepsilon \mu}}=c \sqrt{\left(1+\frac{2 \chi}{c^{2}}\right)}$ [5]; a coordinate system traveling with acceleration $a$ is like an inertial coordinate system traveling in a gravitational field with $\chi$ as the gravitational potential energy, the speed

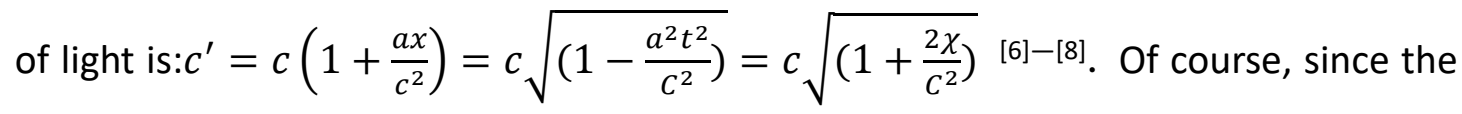
flat space is only for each local locality and instantaneous time, the $x$ and $t$ in the above formulas for the speed of light can only be regarded as valid for each taking a sufficiently small value, in terms of reality, both $a x$ and at can be treated as sufficiently small constants. In short, the speed of light in the instantaneous local inertial coordinate system introduced at each point in the gravitational field (or each instantaneous position of the accelerating particle) is no longer the speed of light $c$ in a vacuum. The values of the speed of light in these instantaneous local inertial coordinate systems $c^{\prime}, c^{\prime \prime}, c^{\prime \prime \prime} \cdots$ actually include the effects of "medium", "gravitational field", "acceleration and deceleration" on the speed of light. Of course, all "weak field", "strong field", "meson field" and even "acceleration" or "deceleration"...and so on may have an impact on the speed of light, and can also be included in the value $c^{\prime}, c^{\prime \prime}, c^{\prime \prime \prime} \cdots$ of the speed of light. This type of inertial coordinate system is the inertial coordinate system with different speed of light as the speed limit determined in [1] [Theorem 5]; If you want to apply the established special theory of relativity to them, you must consider using the established special theory of relativity with variable speed of light $\left(c^{\prime} \neq c\right)$ in [1] and [2]. Looking at the theory of relativity on this basis, there is basically no difference between the general relativity and special relativity. In this way, it is possible to easily and systematically bypass the "mathematical form design task" which cannot be solved completely by the second half of Einstein's life due to the introduction of the " $g_{\mu \nu}$ " in " $d s^{2}=g_{\mu \nu} d x^{\mu} d x^{\nu}$ ", straightforwardly committed to theoretical physics work on the central thesis of relativity.

The coordinate transformation between the instantaneous local inertial coordinate system $S(x, y, z, c t)$ and $S^{\prime}\left(x^{\prime}, y^{\prime}, z^{\prime}, c^{\prime} t^{\prime}\right)$ can also obtain based on the four-dimensional space interval differential line element of remains unchanged in the coordinate transformation, i.e. :

$$
d s^{2}=d x^{2}+d y^{2}+d z^{2}-c^{2} d t^{2}=d x^{\prime 2}+d y^{\prime 2}+d z^{\prime 2}-c^{\prime 2} d t^{\prime 2}=d s^{\prime 2} \quad, \quad\left(c^{\prime} \neq c\right) .
$$

So, we have: 


$$
\begin{array}{rlrl}
d x^{\prime} & =\frac{d x-\mathrm{v} d t}{\sqrt{1-\mathrm{v}^{2} / c^{2}}} & d x & =\frac{d x^{\prime}-\mathrm{v}^{\prime} d t^{\prime}}{\sqrt{1-\mathrm{v}^{\prime 2} / c^{\prime 2}}} \\
d y^{\prime} & =d y & d y & =d y^{\prime} \\
d z^{\prime} & =d z & d z & =d z^{\prime} \\
d t^{\prime} & =\frac{c}{c^{\prime}} \frac{d t-\frac{\mathrm{v}}{c^{2}} d x}{\sqrt{1-\mathrm{v}^{2} / c^{2}}} & d t & =\frac{c^{\prime}}{c} \frac{d t^{\prime}-\frac{\mathrm{v}^{\prime}}{c^{\prime 2}} d x^{\prime}}{\sqrt{1-\mathrm{v}^{\prime 2} / c^{\prime 2}}}
\end{array}
$$

This is exactly the same as the space-time coordinate transformation relationship between inertial systems with different speeds of light as the limit speed obtained in [1], except that the differential representation of the coordinates is used here.

Suppose the speed of a moving particle relative to $S$ and $S^{\prime}$ respectively are:

$$
\vec{u}\left(u_{x}, u_{y}, u_{z}\right)=\left(\frac{d x}{d t}, \frac{d y}{d t}, \frac{d z}{d t}\right) \quad \text { and } \quad \vec{u}^{\prime}\left(u_{x}^{\prime}, u_{y}^{\prime}, u_{z}^{\prime}\right)=\left(\frac{d x^{\prime}}{d t^{\prime}}, \frac{d y^{\prime}}{d t^{\prime}}, \frac{d z^{\prime}}{d t^{\prime}}\right) \text {, }
$$

Then the speed transformation relationship are:

$$
\begin{array}{ll}
u_{x}^{\prime}=\frac{d x^{\prime}}{d t^{\prime}}=\frac{c^{\prime}}{c} \frac{u_{x}-\mathrm{v}}{1-\frac{\mathrm{v} u_{x}}{c^{2}}} & u_{x}=\frac{d x}{d t}=\frac{c}{c^{\prime}} \frac{u_{x}^{\prime}-\mathrm{v}^{\prime}}{1-\frac{\mathrm{v}^{\prime} u_{x}^{\prime}}{c^{\prime 2}}} \\
u_{y}^{\prime}=\frac{d y^{\prime}}{d t^{\prime}}=\frac{c^{\prime}}{c} \frac{u_{y} \sqrt{1-\frac{\mathrm{v}^{2}}{c^{2}}}}{1-\frac{\mathrm{v} u_{x}}{c^{2}}} & u_{y}=\frac{d y}{d t}=\frac{c}{c^{\prime}} \frac{u_{y}^{\prime} \sqrt{1-\frac{\mathrm{v}^{\prime 2}}{c^{\prime 2}}}}{1-\frac{\mathrm{v} u_{x}}{c^{2}}} \\
u_{z}^{\prime}=\frac{d z^{\prime}}{d t^{\prime}}=\frac{c^{\prime}}{c} \frac{u_{z} \sqrt{1-\frac{\mathrm{v}^{2}}{c^{2}}}}{1-\frac{\mathrm{v} u_{x}}{c^{2}}} & u_{z}=\frac{d z}{d t}=\frac{c}{c^{\prime}} \frac{u_{z}^{\prime} \sqrt{1-\frac{\mathrm{v}^{\prime 2}}{c^{\prime 2}}}}{1-\frac{\mathrm{v}^{\prime} u_{x}^{\prime}}{c^{\prime 2}}}
\end{array}
$$

The following derives the functional relationship between the moving mass and the speed.

\section{The jump and stagnation of mass with speed}

The relationship between mass and speed is generally deduced by the collision of two equivalent mass bodies using the law of conservation of momentum. The existence of "equivalence mass bodies" is a basic assumption, and there is no doubt about it. "Collision" is an indispensable minimum method for objective and subjective as well as existence and understanding, there is no need to suspect any man-made interference. The law of conservation of momentum is the most basic law of physics and the main foundation of the theory of relativity. According to the theory of relativity, all the laws of physics have the same mathematical form correctness in any observation coordinate system. Therefore, there is nothing fundamentally suspicious about deriving the mass velocity relationship based on this method.

Suppose two inertial coordinate systems $S$ and $S^{\prime}$, as defined in [Theorem 5] in [1], $S^{\prime}$ can be in the medium, can also be an instantaneous local inertial system of accelerated motion or of in a gravitational field. In $S$ observing $S^{\prime}$ moves at 
speed $\mathrm{v}$ along the $x$ axis of $S$, and the speed of light is $c$; while observing in $S^{\prime}, S$ moves at speed $v^{\prime}$ along the $x^{\prime}$ axis of $S^{\prime}$, the speed of light is $c^{\prime}$. Suppose there are equivalent devices in the $S$ and $S^{\prime}$ systems, each of which shoots an equivalent mass body at the same speed $u$ and $u^{\prime}$ in the direction perpendicular to the motion, and causes them to collide in the relative motion of $S$ and $S^{\prime}$ and then bounce back. This arrangement is realistic, as described in the general relativity monograph ${ }^{[9]}$. However, in the special theory of relativity with variable speed of light, the speed of light in $S$ and $S^{\prime}$ are the unequal $c$ and $c^{\prime}$ respectively, the mass bodies are shot perpendicular to the direction of motion at the same speed and should take care of the basics difference of the space-time metrical unit in $S$ and $S^{\prime}$. That is, the ratio of the speed to the speed of light is equal or unequal, i.e., when $S$ and $S^{\prime}$ are mutually stationary in the speed transposition equation (2) $\frac{u^{\prime}}{c^{\prime}}=\frac{u}{c}\left(\mathrm{v}=\mathrm{v}^{\prime}=0\right)$. This mutual static gives full play to all the equivalent essences of the two equivalent mass bodies, such as the two equivalent atoms each emitting equivalent $\beta$ rays. As for any field or medium surrounding $S$ or $S^{\prime}$ to cause $c^{\prime} \neq c$, it does not damage the equivalence of $S$ and $S^{\prime}$ as inertial coordinate systems. This principle has been elaborated above and in [1] and [2]. Therefore, everything starts from equivalence. In terms of symbols and mathematical forms, equivalence also includes the symmetry between $S$ and $S^{\prime}$. All this is completely in line with the relative viewpoint of relativity theory. It must also be pointed out that the method of cognition will not affect the objective existence, and all the conclusions drawn on the motion mass must exist out of the collision, and will not be changed by other methods of cognition. The choice of collision method to prove, of course, is also based on the fact that this method has no interference from other points.

The subscripts "1" and "2" indicate the mass body in the $S$ and $S^{\prime}$ system respectively; the speeds of the two mass bodies measured in the $S$ and $S^{\prime}$ systems are expressed without "'" and with "'", and a horizontal line is added to the speed symbol to indicate the respective speeds after the collision; then the relationship between the speeds of the two equivalent masses before and after the collision are:

$$
\left.\begin{array}{l}
\bar{u}_{1 x}=u_{1 x}=0, \bar{u}_{1 y}=-\alpha u_{1 y}, \frac{u_{2 y}^{\prime}}{c^{\prime}}=-\frac{u_{1 y}}{c} \\
\bar{u}_{2 x}^{\prime}=u_{2 x}^{\prime}=0, \bar{u}_{2 y}^{\prime}=-\alpha u_{2 y}^{\prime}, \frac{u_{1 y}^{\prime}}{c^{\prime}}=-\frac{u_{2 y}}{c}
\end{array}\right\}
$$

$\alpha$ is a positive number. Apply the speed transformation formula (2) to get:

$$
\left.\begin{array}{l}
\bar{u}_{1 x}^{\prime}=u_{1 x}^{\prime}=\mathrm{v}^{\prime} \\
\bar{u}_{2 x}=u_{2 x}=\mathrm{v}
\end{array}\right\}
$$




$$
\begin{aligned}
& u_{2 y}=\frac{c}{c^{\prime}} u_{2 y}^{\prime} \sqrt{1-\mathrm{v}^{\prime 2} / c^{\prime 2}}=-u_{1 y} \sqrt{1-\mathrm{v}^{\prime 2} / c^{\prime 2}} \\
& u_{1 y}^{\prime}=\frac{c^{\prime}}{c} u_{1 y} \sqrt{1-\mathrm{v}^{2} / c^{2}} \\
& \bar{u}_{2 y}=\frac{c}{c^{\prime}} \bar{u}_{2 y}^{\prime} \sqrt{1-\mathrm{v}^{\prime 2} / c^{\prime 2}}=\alpha u_{1 y} \sqrt{1-\mathrm{v}^{\prime 2} / c^{\prime 2}}=-\alpha u_{2 y} \\
& \bar{u}_{1 y}^{\prime}=-\frac{c^{\prime}}{c} \alpha u_{1 y} \sqrt{1-\mathrm{v}^{2} / c^{2}}
\end{aligned}
$$

Therefore, the square of the eight speed values is calculated as:

$$
\left.\begin{array}{l}
u_{1}^{2}=u_{1 y}^{2} \\
u_{2}^{2}=\mathrm{v}^{2}+u_{1 y}^{2}\left(1-\frac{\mathrm{v}^{\prime 2}}{c^{\prime 2}}\right) \\
u_{1}^{\prime 2}=\mathrm{v}^{\prime 2}+\left(\frac{c^{\prime}}{c}\right)^{2} u_{1 y}^{2}\left(1-\frac{\mathrm{v}^{2}}{c^{2}}\right) \\
u_{2}^{\prime 2}=u_{2 y}^{\prime 2}=\left(\frac{c^{\prime}}{c}\right)^{2} u_{2 y}^{2} \\
\bar{u}_{1}^{2}=\bar{u}_{1 y}^{2}=\alpha^{2} u_{1 y}^{2} \\
\bar{u}_{2}^{2}=\mathrm{v}^{2}+\alpha^{2} u_{1 y}^{2}\left(1-\frac{\mathrm{v}^{\prime 2}}{c^{\prime 2}}\right) \\
\bar{u}_{1}^{\prime 2}=\mathrm{v}^{\prime 2}+\left(\frac{c^{\prime}}{c}\right)^{2} \alpha^{2} u_{1 y}^{2}\left(1-\frac{\mathrm{v}^{2}}{c^{2}}\right) \\
\bar{u}_{2}^{\prime 2}=\bar{u}_{2 y}^{\prime 2}=\left(\frac{c^{\prime}}{c}\right)^{2} \alpha^{2} u_{1 y}^{2}
\end{array}\right\}
$$

Assume that the functional relationship of mass with speed in the $S$ and $S^{\prime}$ systems respectively is $m=f(\mathrm{v})$ and $m^{\prime}=f^{\prime}\left(\mathrm{v}^{\prime}\right)$; two identical masses that are at rest refer to $m_{0}=f(0)=f^{\prime}(0)=m_{0}^{\prime}$. In the $S$ system, the law of conservation of momentum before and after the collision can be written as:

$$
\begin{gathered}
f\left(u_{1}\right) u_{1 x}+f\left(u_{2}\right) u_{2 x}=f\left(\bar{u}_{1}\right) \bar{u}_{1 x}+f\left(\bar{u}_{2}\right) \bar{u}_{2 x} \\
f\left(u_{1}\right) u_{1 y}+f\left(u_{2}\right) u_{2 y}=f\left(\bar{u}_{1}\right) \bar{u}_{1 y}+f\left(\bar{u}_{2}\right) \bar{u}_{2 y}
\end{gathered}
$$

Substituting the relevant quantities in equations (3)-(7) into equations (8) and (9) respectively, we can get:

$$
\begin{aligned}
& f\left(u_{2}\right)=f\left(\bar{u}_{2}\right) \\
& f\left(u_{1 y}\right) u_{1 y}+\alpha f\left(\alpha u_{1 y}\right) u_{1 y}+f\left(u_{2}\right) u_{2 y}+\alpha f\left(\bar{u}_{2}\right) u_{2 y}=0
\end{aligned}
$$

Which is: 


$$
\begin{aligned}
& f\left(\sqrt{\mathrm{v}^{2}+u_{1 y}^{2}\left(1-\mathrm{v}^{\prime 2} / c^{\prime 2}\right)}\right)=f\left(\sqrt{\mathrm{v}^{2}+\alpha^{2} u_{1 y}^{2}\left(1-\mathrm{v}^{\prime 2} / c^{\prime 2}\right)}\right) \\
& f\left(u_{1}\right)+\alpha f\left(\alpha u_{1 y}\right)-\sqrt{1-\mathrm{v}^{\prime 2} / c^{\prime 2}}\left[f\left(u_{2}\right) u_{2 y}+\alpha f\left(\bar{u}_{2}\right)\right]=0
\end{aligned}
$$

If momentum is conserved in any collision of this kind, then for any value of $u, v\left(u^{\prime}, v^{\prime}\right)$, the function $f$ should satisfy equations (10) and (11).

Now take $u_{1 y} \rightarrow 0, \bar{u}_{2}=u_{2}=\mathrm{v}$, on both sides of the formula (10) is naturally satisfied; the formula (11) can be used to calculate $f(v)$; at this time, there is $f(0)-\sqrt{1-\mathrm{v}^{\prime 2} / c^{\prime 2}} f(\mathrm{v})=0$, and the famous formula of traditional special relativity is immediately obtained:

$$
m=f(\mathrm{v})=\frac{f(0)}{\sqrt{1-\mathrm{v}^{\prime 2} / c^{\prime 2}}}=\frac{m_{0}}{\sqrt{1-\mathrm{v}^{\prime 2} / c^{\prime 2}}}
$$

Because when $u_{1 y}=0, \bar{u}_{2}=u_{2}=\mathrm{v}, \mathrm{v}$ in $f(\mathrm{v})$ is the speed of moving mass 2 recognized by observer in the $S$ system, and $v^{\prime}$ is the speed of the observer of $S$ system recognized reversely in the $S^{\prime}$ system attached to the mass. In formula (12), $\mathrm{v}^{\prime 2} / c^{\prime 2}$ is still retained in the denominator square root, which can fully demonstrate the equivalent active effect of cognition and being recognized, showing that $m=f(\mathrm{v})$ must be determined by at least one collision. If more attention is paid to the objective existence of the relationship between mass and speed which does not vary depending on the method of recognizing, then $\mathrm{v}^{\prime 2} / c^{\prime 2}=\mathrm{v}^{2} / c^{2}$ can be applied, and $\mathrm{v}^{2} / c^{2}$ can be used to replace $\mathrm{v}^{\prime 2} / c^{\prime 2}$. In order to avoid confusion with the relative speed between $S$ and $S^{\prime}$, formula (12) is best written as:

$$
m=f(u)=\frac{f(0)}{\sqrt{1-u^{2} / c^{2}}}=\frac{m_{0}}{\sqrt{1-u^{2} / c^{2}}}
$$

It is important that: the symmetrical side of this collision, that is, in the $S^{\prime}$ system, we can also obtain:

$$
m^{\prime}=f^{\prime}\left(\mathrm{v}^{\prime}\right)=\frac{f^{\prime}(0)}{\sqrt{1-\mathrm{v}^{2} / c^{2}}}=\frac{m_{0}^{\prime}}{\sqrt{1-\mathrm{v}^{2} / c^{2}}}
$$

Or

$$
m^{\prime}=f^{\prime}\left(u^{\prime}\right)=\frac{f^{\prime}(0)}{\sqrt{1-u^{\prime 2} / c^{\prime 2}}}=\frac{m_{0}^{\prime}}{\sqrt{1-u^{\prime 2} / c^{\prime 2}}}
$$

Consider the preconditions $m_{0}=m_{0}^{\prime}$, comparing (12a) with (12c) proves: 


$$
m^{\prime}=f^{\prime}\left(u^{\prime}\right)=f(u)=m
$$

It must be understood that here $f^{\prime}=f$ means that the two functions have the same form; it does not mean that the masses measured by the systems $S$ and $S^{\prime}$ at the same instant and the same place of the equivalent mass body are equal. Only when $\frac{u^{2}}{c^{2}}=\frac{u^{\prime 2}}{c^{\prime 2}}$, we have $m=m^{\prime}$

It is especially worth noting that in the above derivation process, $u_{1 y} \rightarrow 0$ is taken first, so $\alpha$ in equations (10) and (11) can be any value. However, if we face (10) first, and pre-determine that $f(u)$ must be a continuous increasing function of speed, we must determine that $\alpha=1, \alpha=1$ are completely elastic collision. This is enough to inspire people to find solutions other than (12); just give up $\alpha=1$ and give up the continuous increasing function form of $f(u)$. According to Newton's classical mechanics, $f(u)$ is a constant to the speed $u$ and does not change with the speed, nor does it continuously increase with the speed, nor will it decrease. Combining the two forms, it is reasonable to take $f(u)$ as a monotonic non-decreasing function form interrupted at a finite (or mathematically "countable") point, that is, a step function. The theory of relativity can thus enter the category of quantum theory as a guiding theory.

Let $f$ be a step function, namely $f\left(u_{2}\right)=f\left(u_{2}+k\right)=f\left(\bar{u}_{2}\right), \quad k$ is a constant with velocity dimension; then the formula (10) becomes:

$$
f\left(\sqrt{\mathrm{v}^{2}+u_{1 y}^{2}\left(1-\mathrm{v}^{\prime 2} / c^{\prime 2}\right)}+k\right)=f\left(\sqrt{\mathrm{v}^{2}+\alpha^{2} u_{1 y}^{2}\left(1-\mathrm{v}^{\prime 2} / c^{\prime 2}\right)}\right)
$$

Thus:

$$
\sqrt{\mathrm{v}^{2}+u_{1 y}^{2}\left(1-\mathrm{v}^{\prime 2} / c^{\prime 2}\right)}+k=\sqrt{\mathrm{v}^{2}+\alpha^{2} u_{1 y}^{2}\left(1-\mathrm{v}^{\prime 2} / c^{\prime 2}\right)}
$$

After squaring (13) and shifting the terms, we get:

$$
k^{2}+2 k \sqrt{\mathrm{v}^{2}+u_{1 y}^{2}\left(1-\mathrm{v}^{\prime 2} / c^{\prime 2}\right)}=\left(1-\mathrm{v}^{\prime 2} / c^{\prime 2}\right) u_{1 y}^{2}\left(\alpha^{2}-1\right)
$$

In order to be able to eliminate $\alpha$ and get $k$ under other conditions with known values, a similar equation is needed. Now the equal symmetry condition of $S$ and $S^{\prime}$ is fully functional. In the $S^{\prime}$ system, it has been proved that $f^{\prime}=f$; due to equality, $f^{\prime}$ and $f$ must have the same values of $k$ and $\alpha$; according to the equivalent arrangement, $u_{2 y}^{\prime}$ in the $S^{\prime}$ system is equivalent to $u_{1 y}$ in the $S$ system, and 
$u_{2 y}^{\prime}=-\left(\frac{c^{\prime}}{c}\right) u_{1 y}$, so it can be deduced immediately in the $S^{\prime}$ system:

$$
k^{2}+2 k \sqrt{\mathrm{v}^{\prime 2}+\left(\frac{c^{\prime}}{c}\right)^{2} u_{1 y}^{2}\left(1-\mathrm{v}^{2} / c^{2}\right)}=\left(1-\mathrm{v}^{2} / c^{2}\right)\left(\frac{c^{\prime}}{c}\right)^{2} u_{1 y}^{2}\left(\alpha^{2}-1\right)
$$

Note that $\frac{\mathrm{v}^{2}}{c^{2}}=\frac{\mathrm{v}^{\prime 2}}{c^{\prime 2}}$, divide (14) and (14a), and after simple calculation, we can get:

$$
k=-\frac{2 c^{\prime}}{c+c^{\prime}} \sqrt{\mathrm{v}^{2}+u_{1 y}^{2}\left(1-\mathrm{v}^{2} / c^{2}\right)}
$$

It is easy to see that $k$ obtained from the $S^{\prime}$ system of symmetry is also equal to the above formula. It must be pointed out that in the case of the traditional special theory of relativity $c^{\prime}=c$, the two equations (14) and (14a) are exactly the same, and it is still impossible to eliminate $\alpha$ to solve $k$; therefore, even if the trial of entering the step function is still forced to exit. The method of traditional special relativity is limited, this is an important key example.

Now, take $f\left(u_{2}\right)=f\left(\bar{u}_{2}\right)=f\left(\sqrt{\mathrm{v}^{2}+u_{1 y}^{2}\left(1-\mathrm{v}^{2} / c^{2}\right)}+k\right)$ and enter (11) again, and still set $u_{1 y} \rightarrow 0$, then:

$$
(1+\alpha) f(0)=(1+\alpha) \sqrt{1-\mathrm{v}^{\prime 2} / c^{\prime 2}} f\left(\frac{c-c^{\prime}}{c+c^{\prime}} \mathrm{v}\right)=(1+\alpha) \sqrt{1-\mathrm{v}^{\prime 2} / c^{\prime 2}} f(\mathrm{v})
$$

From this, the step function relationship of mass with speed is obtained:

$$
f\left(\frac{c-c^{\prime}}{c+c^{\prime}} \mathrm{v}\right)=\frac{m_{0}}{\sqrt{1-\mathrm{v}^{2} / c^{2}}}=f(\mathrm{v})
$$

For the first half of equation (16), let $\left(\frac{c-c^{\prime}}{c+c^{\prime}}\right) \mathrm{v}=u$, then $\mathrm{v}=\left(\frac{c+c^{\prime}}{c-c^{\prime}}\right) u$, the first half of the equation becomes:

$$
f(u)=\frac{m_{0}}{\sqrt{1-\frac{u^{2}}{c^{2}}\left(\frac{c+c^{\prime}}{c-c^{\prime}}\right)^{2}}}
$$

The step function relationship between mass and speed represented by (16) and (16a) is shown in (Fig. 1).

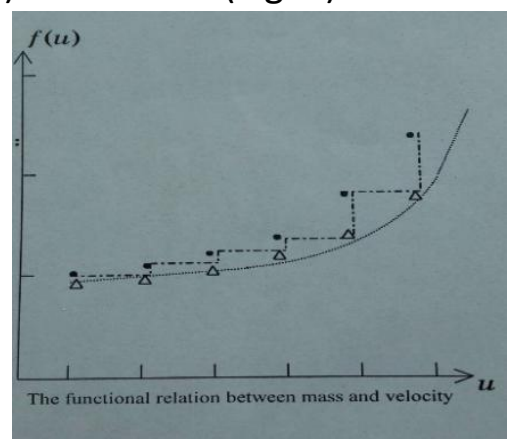

(Fig.1) 
"•" in the figure represents the first half of (16) and (16a); " $\triangle$ " represents the second half of formula (16). It can be seen from equation (16) and Fig.1: For the same mass $m=\frac{m_{0}}{\sqrt{1-\mathrm{v}^{2} / c^{2}}}$, there can be two different speeds, $u_{1}=\left(\frac{c-c^{\prime}}{c+c^{\prime}}\right) \mathrm{v}(" \bullet "$ in the Fig.1) and $u_{2}=\mathrm{v}$ (" $\triangle$ " in the Fig.1), such as one step extension forward on the same step stair. On the other hand, comparing the latter half of Eq. (16) with Eq. (16a), we see that when $\mathrm{v}=u$, for the same speed $u$, the mass can have two different values, $m_{1}=\frac{m_{0}}{\sqrt{1-\frac{u^{2}}{c^{2}}}}\left(" \triangle "\right.$ in the Fig.1) and $m_{2}=\frac{m_{0}}{\sqrt{1-\frac{u^{2}}{c^{2}}\left(\frac{c+c^{\prime}}{c-c^{\prime}}\right)^{2}}}(" \bullet "$ in the Fig.1), such as a ladder upgrade one level. From the perspective of the increase in speed, the mass is stagnant on the step platform (the speed increases, the mass does not change), and it jumps in the step up ladder (the speed does not change, the mass changes suddenly). The calculation shows that when the speed gradually increases, the steps gradually lengthen and the stair level gradually increase. This is a kind of discontinuous step jump change relationship of mass with the speed, which is different from the continuous incremental change relationship known in the traditional special theory of relativity.

It should be noted that although the second half of equation (16) is the same as the traditional solution (12), these two types of solutions do not exist at the same time. To prove this conclusion, substituting formula (15) into formula (13), we can find $\alpha$ to get:

$$
\alpha^{2}=\left(\frac{c-c^{\prime}}{c+c^{\prime}}\right)-\frac{\mathrm{v}^{2}\left[1-\left(\frac{c-c^{\prime}}{c+c^{\prime}}\right)^{2}\right]}{u_{1 y}^{2}\left(1-\frac{\mathrm{v}^{2}}{c^{2}}\right)}
$$

It can be seen from equation (17) that in the above step function calculation, the value of $\alpha$ cannot be equal to 1 ; $\alpha=1$ is only suitable for solving traditional continuous functions. Therefore, this important conclusion must be emphasized: the two types of solutions of the mass velocity relationship ,that is, the discontinuous step function and the continuous increasing function will not coexist at the same time; Equation (16) and Equation (16a), or the discontinuous step function solution shown in Fig.1, includes many " $\triangle$ " values equal to the value of the traditional continuous function calculation equation (12), but the former is not allowed $\alpha=1$, so they will never exist at the same time. The traditional continuous function solution is to connect the many values at " $\triangle$ " with a curve (the dotted curve in the Fig.1); the discontinuous step function solution connects the horizontal step platform at the "•" and " $\triangle$ " (the dotted horizontal line in the Fig.1) is based on The assumption that the quality does not decrease due to the increase in speed. 
Add some important conclusions to the formula derived above.

(1) Because vis a real number, the maximum value of $|\alpha|$ given by (17) is $\left|\frac{c-c^{\prime}}{c+c^{\prime}}\right|$, and the minimum value is zero. One situation of $|\alpha|=0$ is $c=c^{\prime}, \mathrm{v}=0$, $\frac{\mathrm{v}^{2}\left(c+c^{\prime}\right)^{2}}{c^{2}\left(c-c^{\prime}\right)^{2}}$ is an indefinite value, that is, $m$ and $m_{0}$ have no definite relationship. The meaning of $|\alpha|=0$ means that the two particles are bonded after the collision, which is completely inelastic collision. Therefore, in R. A. Millikan's first experiment to measure the electron-to-mass ratio $\frac{e}{m}$ in the cloud chamber, there should be several $e$ in each floating oil drop, not limited to one $e$; in fact, all of them meet $c=c^{\prime}, \quad \mathrm{v}=0,|\alpha|=0$ explanation. This is actually a major aspect to be considered when interpreting material reunion.

(2) For the solution of the step function, it can be seen from equation (16a) that the upper limit speed of the constant velocity moving particle is $\tilde{u}=\frac{c-c^{\prime}}{c+c^{\prime}} c<c$, and when the upper limit speed is reached, $m_{0}=0, m=f(\tilde{u})$ is an indeterminate. Because the electron's $m_{0} \neq 0$, the actual velocity of the $\beta$-ray can never reach $\tilde{u}$, which is less than the traditional saying that it is less than $c$. Extrapolate from the experimental data to find the $\tilde{u}$ of a certain type of $\beta$-ray, and then the value of $c^{\prime}$ at the point where the $\beta$-ray starts can be calculated. This should advance the theory of relativity inside the atom.

(3) When the values of $c$ and $c^{\prime}$ are very close, that is, when $\left(c-c^{\prime}\right) \rightarrow 0$, the upper speed limit $\tilde{u}$ also approaches zero. At this time, we return to the inference in (1) above, and explain that when $|\alpha|=0$ other solutions of equation (17) are unrealistic. When the speed is very small, relativistic mechanics approaches classical mechanics, so the solution of the step function is also the same. From the tiny value of $\pm\left(\frac{c-c^{\prime}}{c+c^{\prime}}\right)$, one can imagine the shortening of steps and the dropping of stairs in Fig. 1 , that is, the step function curve gradually approaches the smooth curve of traditional solution. This also explains why Newtonian mechanics is extremely realistic when $c=c^{\prime}$. 
(4) When the values of $c$ and $c^{\prime}$ differ greatly, such as $c: c^{\prime}=100: 1,\left(\frac{c-c^{\prime}}{c+c^{\prime}}\right)$ is very close to 1 and only slightly less than 1 , such as $\frac{99}{101}$. This in turn makes the step curve close to the smooth curve of the traditional solution. Very small $c^{\prime}$ means that light is difficult to penetrate, which is similar to the situation in which thermionic electrons are driven out of the cathode surface; it is conceivable that these thermionic electrons are difficult to show a stepped mass-velocity relationship curve.

(5) When $c^{\prime}$ is closer to the middle value between zero and $c$, the stepped massvelocity relationship curve is more obvious. This can only be discovered on the ejected mass inside the atom.

In the special theory of relativity with variable speed of light, the step function relationship of the discontinuous change of mass with speed and its related conclusions have opened the door for the theory of relativity to enter quantum theory. A schematic sketch has been prepared for the theory of relativity dominating modern physics. In atoms fields and various particles fields, the speed of light is definitely not a constant value $c$, The key is to grasp the meaning of the non-coexistence of the traditional continuous function solution $\left(\alpha^{2}=1\right)$ and the step function solution $\left(\alpha^{2} \neq 1\right)$, and the fact that the two solutions are very close when $c^{\prime} \rightarrow 0$ or $c^{\prime} \rightarrow c$, and further study in combination with the actual situation. First of all, it is to seek the proper combination of mass, energy and momentum allowed by the "ontology" of the moving mass bodies in various stages of motion properties or in different physical environments.

\section{acknowledgement}

This article is based on Professor Dong Zhonglin's research work on the theory of relativity from 1965 to 1976 .

Dong Zhonglin (1907-1976), one of the second batch international students in 1934 that scholarship supported by the Gengzi Indemnity of Sino-British. Received a doctorate degree from Cornell University in 1937. He has taught at Northwest Associated University, Guangxi University, Fudan University, Nanjing University, Tongji University, Wuhan Institute of Surveying and Mapping and other institutions. Unfortunately, he died of a sudden cardiac infarction in his residence at Fudan University on April 27, 1976. With deep memory and admiration for Mr. Dong Zhonglin, the author of this article releases the research results to commemorate this forefather's pursuit and exploration of scientific truth.

\section{References}

[1] Dong Jun, Na Dong, The special theory of relativity in different media( I ) https://www.researchsquare.com/article/rs-403193/v1 
[2] Na Dong, Dong Jun, The special theory of relativity in different media ( II ) https://www.researchsquare.com/article/rs-403773/v1

DOI: $10.21203 /$ rs.3.rs-403773/v1

[3] C. mфller, The Theory of Relativity , [M] , London : Oxford Press .,1952 , 240

[4] R. S. Shankland, 1963 Am. J. Phys. 31, 47

[5] C. mфller, The Theory of Relativity , [M] , London : Oxford Press .,1952 , 308

[6] C. mфller, The Theory of Relativity , [M] , London : Oxford Press ., 1952 , 250-258

[7] Wu Dayou, Li Rongzhang , Inter. Jour. Theoretical Physics 5,(1972),307-323

[8] Wu Dayou, Theory of Relativity, Theoretical Physics Volume 4, [M], Beijing: Science Press, 1983, 172-175

[9] C. mфller, The Theory of Relativity , [M] , London : Oxford Press ., 1952, 67

[10] P. G. Bergmann , Introduction to the Theory of Relativity ,[M] New York: PrenticeHall, 1946, 92 


\section{Figures}



\section{Figure 1}

The step function relationship between mass and speed represented by (16) and (16a) 